\title{
Efeito da Hibridização de Montmorilonita e Celulose II sobre as Propriedades Mecânicas de Nanocompósitos de Borracha Natural
}

\author{
Roberta M. Mariano, Regina C. R. Nunes, Leila L. Y. Visconte \\ Universidade Federal do Rio de Janeiro - UFRJ, Instituto de Macromoléculas Professora Eloísa Mano
}

Volker Altstaedt

Department of Polymer Engineering, University of Bayreuth, Universitaetsstr

\begin{abstract}
Resumo: Nanocompósitos híbridos de borracha natural (NR) com argila mineral montmorilonita (MMT) e celulose II (CEL II) foram obtidos partindo de látex elastomérico, xantato de celulose e suspensão aquosa de MMT. O conteúdo de MMT foi variado de 1 a 5 phr (per hundred resin) e a quantidade de celulose II utilizada foi mantida constante em $15 \mathrm{phr}$. Foi comprovada por microscopia eletrônica de transmissão (TEM) a formação de nanocompósitos, na ausência e presença de xantato de celulose, mostrando a eficiência da técnica utilizada. Além do resultado de TEM, a viscosidade e relaxação Mooney, e propriedades mecânicas foram estudadas em todas as composições desenvolvidas. A adição da argila mineral promoveu aumento nas propriedades mecânicas estudadas, sendo que o aumento na viscosidade Mooney e a redução na capacidade de relaxação indicaram boa interação entre a montmorilonita e o sistema NR/CEL II.

Palavras-chave: Nanocompósito híbrido, látex de borracha natural, argila, celulose.

\section{Effect of Montmorillonite and Cellulose II Hybridization on Mechanical Properties of Natural Rubber Nanocomposites}

\begin{abstract}
Hybrid nanocomposites of natural rubber (NR), clay (MMT) and cellulose II (CEL II) were obtained with elastomeric latex, cellulose xanthate and aqueous suspension of the clay. The content of MMT varied from 1 to 5 phr (per hundred resin) while cellulose II was kept as $15 \mathrm{phr}$. The formation of nanocomposites from co-coagulation of natural rubber latex and MMT aqueous suspension, in the presence and absence of cellulose xanthate, was confirmed by transmission electron microscopy (TEM), thus showing the efficiency of the technique used. In addition to the TEM investigation, all compositions were studied with measurements of the Mooney viscosity and relaxation and mechanical properties. The MMT addition improved the mechanical properties, causing an increase in Mooney viscosity and a reduction in relaxation capability, which indicate a good interaction between MMT and NR/CEL II system.
\end{abstract}

Keywords: Hybrid nanocomposite, natural rubber latex, clay, cellulose.

\section{Introdução}

A borracha natural é um polímero que apresenta propriedades que não são tecnicamente reproduzidas nos elastômeros sintéticos. A facilidade de recuperação rápida após cessada a deformação, a capacidade de suportar a aplicação de níveis elevados de carga devido a elasticidade, elevada resistência à abrasão e ao impacto e elevada resistência a choques térmicos fazem da borracha natural um elastômero com comportamento peculiar apresentando também elevada demanda industrial e comercial, onde os pneus são os principais representantes comerciais ${ }^{[1]}$.

As cargas são adicionadas à matriz elastomérica para promover o reforço, sendo o negro de fumo e a sílica as cargas mais utilizadas comercialmente. Com o advento da nanotecnologia, a incorporação de outras substâncias tem promovido melhora nas propriedades das matrizes poliméricas. Devido ao tamanho nanométrico das partículas de carga dispersas nas matrizes poliméricas, os nanocompósitos desenvolvem comportamentos particulares e elevadas propriedades quando comparados aos compósitos tradicionais. O tamanho máximo das partículas de carga presentes nos materiais nanoestruturados é de $100 \mathrm{~nm}$ e estes permitem a incorporação de nano cargas em quantidades relativamente pequenas para a obtenção de um bom desempenho estrutural $^{[2]}$.

A adição de cargas inorgânicas reforçadoras ao sistema polimérico leva a formação de compósitos híbridos. O termo híbrido vem do grego hybris que significa mistura ou associação de diferentes espécies ou substâncias ${ }^{[3]}$. O uso de argilominerais como carga de reforço formando compósitos híbridos apresenta vantagens como altas resistências químicas e mecânicas e estabilidade térmica. Nesse tipo de material híbrido, propriedades como alta resistência mecânica e dureza, e menor permeabilidade a gases são alcançadas usando uma menor quantidade de material inorgânico da que é usada nos compósitos poliméricos convencionais com carga ${ }^{[4,5]}$.

A celulose II ou celulose regenerada, obtida da coagulação do xantato de celulose, é uma das formas alomórficas da celulose nativa, também denominada celulose I. A partir do processo de mercerização, a celulose I extraída da polpa da madeira é dissolvida em hidróxido de sódio. Após este processo, ocorre a adição de dissulfeto de carbono formando uma solução de cor alaranjada chamada de xantato de celulose, que é novamente dissolvido em hidróxido de sódio. O xantato de celulose é o precursor do celofane e do raion viscose $\mathrm{e}^{[6,7]}$.

O processo utilizado por este grupo de pesquisa para o desenvolvimento de nanocompósitos elastoméricos de borracha com CEL II ${ }^{[8-12]}$ permite que dois polímeros, um amorfo (NR) e outro extremamente cristalino (CEL II), formem um nanomaterial que apresenta bom desempenho mecânico e impermeabilidade a gases ${ }^{[11,12]}$. Este processo é baseado na co-coagulação do látex elastomérico com solução alcalina de xantato de celulose $\mathrm{e}^{[9]}$. 
Considerando que a adição da celulose $\mathrm{II}^{[8-12]}$, ou da montmorilonita ${ }^{[13-15]}$, separadamente na forma nanométrica, promovem um bom desempenho nas propriedades da borracha natural, o objetivo deste trabalho foi o estudo do efeito da incorporação de MMT na borracha natural com e sem celulose II (CEL II), em relação às propriedades mecânica e morfológica, na tentativa de uma avaliação quanto a interações MMT-NR e MMR-NR/CEL II.

A quantidade de celulose II utilizada para obtenção de todas as composições foi mantida constante em $15 \mathrm{phr}$ (per hundred resin) devido à obtenção de melhor desempenho mecânico observado em trabalhos anteriores ${ }^{[8,9,11,12]}$ e o conteúdo de MMT foi variado de 1 a $5 \mathrm{phr}$. As contribuições deste trabalho se referem a caracterização dos nanocompósitos desenvolvidos quanto a morfologia por difração de raio-X, TEM, as determinações da viscosidade e relaxação Mooney, a análise das propriedades mecânicas como deformação permanente por compressão (DPC), resiliência e resistência à tração sob tensão. Pelos resultados gráficos de tensão versus deformação foi possível a análise da cristalização sob tensão da NR indicando interações entre a MMT e a borracha natural, e entre a MMT e o sistema NR/CEL II.

\section{Experimental}

\section{Materiais}

O látex de borracha natural com $61,9 \%$ de sólidos totais foi doado pela indústria Comércio e Beneficiamento de Látex Talismã Ltda. - São Paulo, Brasil; a argila montmorilonita sódica (Volclay SPV 200) com origem em Wyoming, EUA, foi fornecida pela Bentonit União Nordeste S/A e o xantato de celulose (com 9,8\% de celulose II) foi cedido pela Vicunha Têxtil S/A, São Paulo, Brasil. Todos os materiais e os aditivos da formulação de NR e foram usados como recebidos.

\section{Preparo dos nanocompósitos}

Os nanocompósitos foram obtidos pela dispersão da argila em água (no teor de 1 a 5 phr) sob vigorosa agitação, seguida da adição desta dispersão ao látex de NR. Após a homogeneização da mistura, o xantato de celulose foi adicionado sob agitação, e a mistura foi submetida ao processo de co-coagulação com solução tampão ácida. Os nanocompósitos foram lavados, secos e as composições NR/MMT com ou sem CEL II, foram submetidas ao misturador de rolos aberto com razão de velocidade de 1:1,25, de acordo com a ASTM D 3182, a temperatura ambiente, para a obtenção da composição vulcanizável. A formulação usada foi a seguinte, (em phr): $\mathrm{NR}=100 ; \mathrm{ZnO}=3,5 ;$ Ácido esteárico = 2,5; Enxofre = 2,5; CBS (N-cicloexil-2-benzotiazolsulfenamida) $=0,8$; Aminox (produto obtido da reação a baixa temperatura, entre acetona e difenilamina) $=2,0$; Celulose $\mathrm{II}=15 ; \mathrm{MMT}=1$ a 5 .

$\mathrm{O}$ detalhamento desta parte experimental encontra-se descrito em uma publicação recente ${ }^{[16]}$.

\section{Caracterização}

\section{Propriedades mecânicas}

O ensaio de resistência à tração foi realizado em máquina universal modelo EMIC, de acordo com a norma DIN 53504. O ensaio de deformação permanente após a compressão foi realizado de acordo com a norma ASTM D 395, com os corpos de prova em duplicata. A resiliência das composições foi avaliada segundo a ASTM D 1054.

\section{Difração de raio-X a altos ângulos}

A difração de raios- $X$ foi realizada com radiação de $\mathrm{Cu} \mathrm{K} \alpha$ $\left(\lambda_{\mathrm{Cu}}=1,54 \AA\right.$ ) e velocidade do goniômetro de $0,5^{\circ}, 2 \theta$ por passo. A
Lei de Bragg $(n \lambda=2 d \sin \theta)$ foi utilizada para calcular o espaçamento cristalográfica $(\mathrm{d})$

\section{Microscopia eletrônica de transmissão (TEM)}

A microscopia eletrônica de transmissão foi realizada em microscópio Zeiss $902 \mathrm{~A}$ utilizando voltagem de $80 \mathrm{kV}$. As amostras foram preparadas por crio-ultramicrotomia a $-110{ }^{\circ} \mathrm{C}$, utilizando um ultramicrotomo modelo Keichert-Jung com faca modelo Ultracut E, com velocidade de corte de $0,1 \mathrm{~mm} / \mathrm{s}$ e espessura de aproximadamente $60 \mathrm{~nm}$.

\section{Viscosidade e relaxação Mooney}

A viscosidade e relaxação de todas as composições obtidas foram analisadas utilizando o viscosímetro Mooney MV2000 Alpha Technologies a $100{ }^{\circ} \mathrm{C}$ de acordo com ASTM D 1646 utilizando rotor grande (ML). A relaxação Mooney (MR) é definida de acordo com a Equação 1.

$$
M R=\left[\frac{M 30}{M L(1+4)}\right] \times 100
$$

onde $\mathrm{M}_{30}$ é a viscosidade Mooney medida 30 segundos após a parada do rotor, isto é, após ML (1+4) ser determinado, de acordo com ASTM D 1646.

\section{Resultados e Discussão}

A morfologia dos nanocompósitos de NR/CEL/MMT foi estudada a partir das análises de difração de raio-X e micrografia obtida por microscopia eletrônica de transmissão (TEM), que fornece imagens sobre a dispersão e distribuição da argila e da celulose na matriz de NR. A Figura 1 apresenta os difratogramas dos nanocompósitos de borracha natural com argila (a) e borracha natural com argila e celulose II. As análises de difração de raio-X revelaram que as composições NR/1MMT, NR/2MMT e NR/3MMT não apresentaram pico de difração. A distância entre os planos cristalográficos referente ao pico da montmorilonita é de 1,36 nm. As composições NR/4MMT e NR/5MMT apresentaram distância interplanares de $1,85 \mathrm{~nm}$ e $1,44 \mathrm{~nm}$, respectivamente. De acordo com a literatura, a ausência e intensa redução do pico de difração da MMT nas composições sugerem a intercalação/esfoliação das lamelas de $\mathrm{MMT}^{[17,18]}$. A adição de celulose ao sistema borracha natural/MMT levou ao total desaparecimento dos picos de difração não permitindo a identificação da quantidade limite de MMT para intercalação e/ou esfoliação das lamelas do argilomineral na mistura NR/CEL/MMT na faixa analisada.

A Figura 2 apresenta a micrografia de TEM para a borracha natural com 15 phr de celulose II e 2 phr de MMT. A Figura 2 possibilita a observação da morfologia da celulose II e confirmam a presença de nanocelulose presente na matriz de borracha natural ${ }^{[19]}$. As linhas escuras na imagem correspondem às interseções das camadas do silicato. Alguns pontos que aparecem próximos a estas linhas correspondem à celulose II dispersa na matriz. Pode ser observado que as lamelas de argila apresentaram espessura muito fina e são encontrados domínios de agregados/intercalados/esfoliados em diferentes direções do argilo mineral na matriz borrachosa. Esta micrografia confirma a formação de nanocompósitos e corroboram os resultados de difração de raios-X.

Desta forma, a técnica de co-coagulação mostrou-se efetiva e viável para a fabricação de nanocompósitos, partindo de látex elastomérico. É importante acrescentar que na produção industrial de borracha sólida, advinda de polimerização por emulsão, a técnica usada é similar, usando a coagulação do látex, lavagem dos grânulos obtidos e secagem, podendo desta forma o processamento, na obtenção dos nanocompósitos borracha/celulose/MMT ser proposto em escala industrial. 


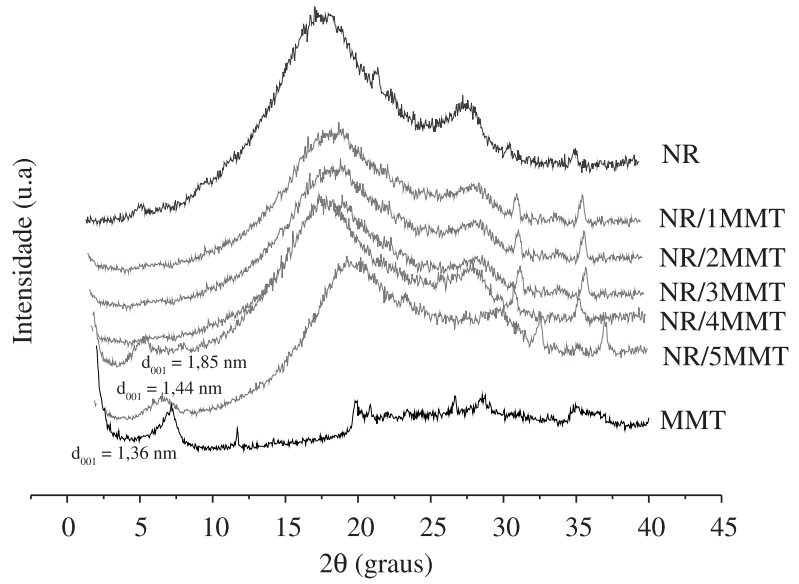

(a)

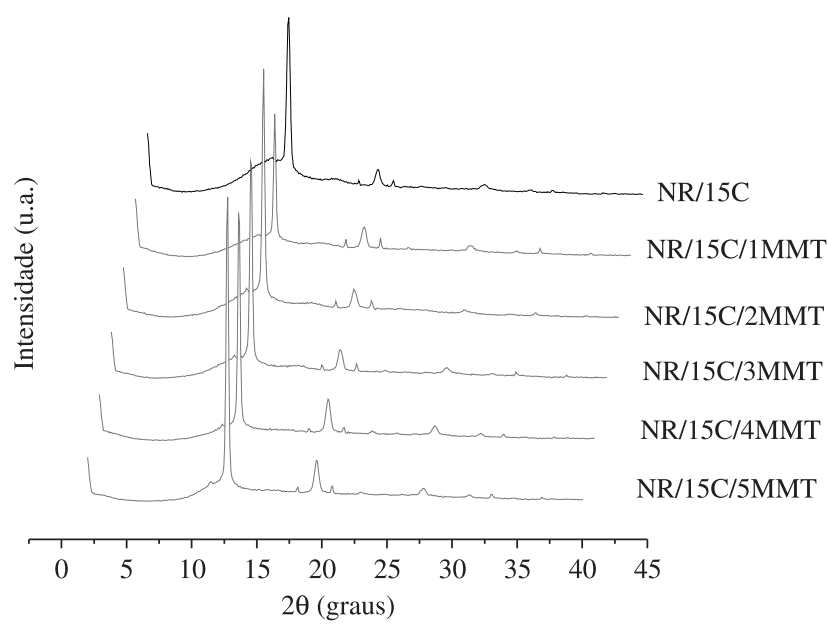

(b)

Figura 1. Difratogramas dos nanocompósitos de borracha natural com argila (a) e borracha natural com argila e celulose II.

A Figura 3 apresenta os resultados de viscosidade e relaxação Mooney dos nanocompósitos de borracha natural determinados a $100{ }^{\circ} \mathrm{C}$. Pode ser observado que a adição de celulose a borracha goma pura provocou um pequeno aumento na viscosidade Mooney e que este aumento foi bem significativo com a adição de argila ao sistema NR/CEL até $3 \mathrm{phr}$ de MMT. Este comportamento pode ser atribuído a boa dispersão da argila na matriz elastomérica e/ou a interações entre as lamelas de argila intercalada/esfoliada e o sistema NR/CEL ${ }^{[17]}$. Os resultados de viscosidade Mooney corroboram os resultados reométricos previamente obtidos ${ }^{[16]}$.

A relaxação Mooney é uma medida da taxa de recuperação das cadeias de borracha submetidas à deformação cisalhante aplicada durante a análise. De acordo com os resultados, a rigidez do nanopolímero celulose e a adição de argila dificultam o processo de relaxação das cadeias de borracha após cessada a deformação cisalhante aplicada. Este fato pode ser atribuído ao confinamento das cadeias de NR/CEL entre as lamelas de montmorilonita. O aumento da viscosidade e a redução da capacidade de relaxação podem ser observados até $3 \mathrm{phr}$ de montmorilonita presentes no sistema elastomérico. Após este conteúdo, a viscosidade diminui e a capacidade de relaxação, e recuperação elástica aumenta indicando a formação de agregados de carga.

O desempenho dos nanocompósitos de borracha natural com celulose II e montmorilonita foram estudados com base em alguns ensaios mecânicos, tradicionalmente realizados na indústria de borracha. As características da carga de reforço em elastômeros, assim como teor, estrutura, tamanho, área superficial e grau de dispersão na matriz elastomérica exercem grande influência nas propriedades mecânicas dos compostos de borracha ${ }^{[1]}$.

As curvas de tensão versus deformação são de grande interesse na análise da influência de cargas na cristalização sob tensão de NR, cujos resultados estão diretamente ligados ao desempenho final do $\operatorname{artefato}^{[20,21]}$.

O comportamento mecânico dos nanocompósitos de borracha natural sem e com celulose, variando o conteúdo de montmorilonita, pode ser observado a partir das curvas obtidas no ensaio de Tensão de tração, mostradas na Figura 4. Na Figura 4a é constatado que a adição de argila à matriz elastomérica provocou aumento da resistência à tração das composições até o conteúdo de $3 \mathrm{phr}$ de MMT. Entretanto, com a adição de celulose à matriz elastomérica, o melhor desempenho mecânico sob tração foi obtido com 2 phr de MMT, como ilustrado na Figura 4b. A adição do nanopolímero celulose II promoveu um aumento na resistência à tração das composições devido às características próprias da celulose, como cristalinidade e rigidez molecular, e também devido a sua dispersão e da MMT na matriz de NR. Estes

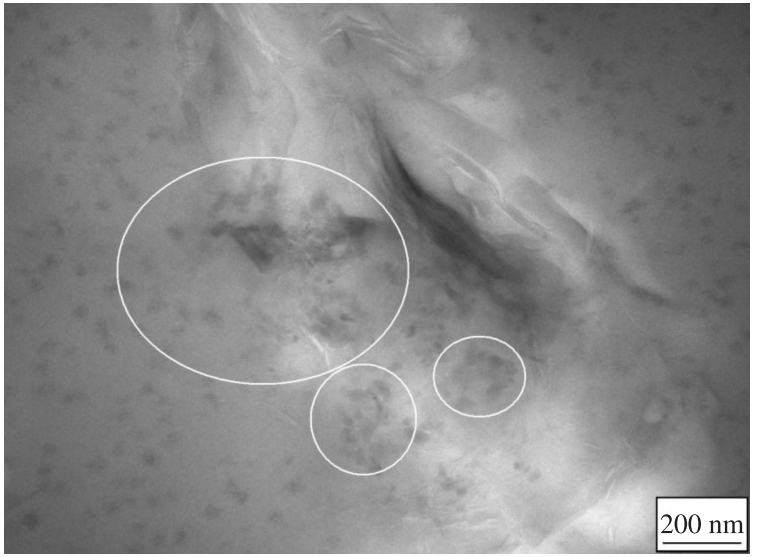

Figura 2. Micrografia de microscopia eletrônica de transmissão (TEM) de borracha natural com celulose II e 2 phr de montmorilonita.

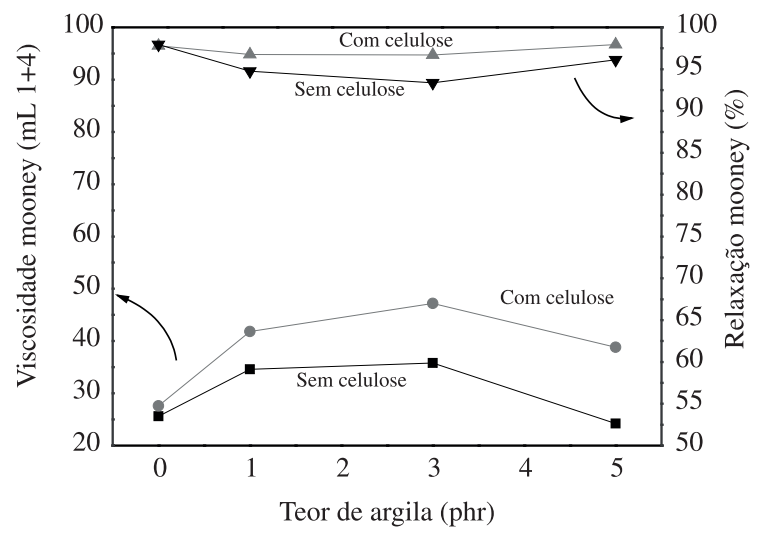

Figura 3. Viscosidade e relaxação Mooney dos nanocompósitos de NR.

resultados corroboram os valores encontrados para a viscosidade e a relaxação Mooney, e também das propriedades reométricas mostradas em trabalho anterior ${ }^{[16]}$, e são consequência da intercalação/esfoliação de montmorilonita até os teores de MMT citados.

Segundo $\mathrm{Vu}$ et al., as cadeias de borracha encontram-se alocadas na superfície das lamelas intercaladas/esfoliadas de MMT. Considerando que as lamelas intercaladas/esfoliadas apresentam elevada área superficial, o aumento no conteúdo de argila permite que uma boa quantidade de cadeias de borracha seja absorvida pelas lamelas, resultando em baixa capacidade de deformação ${ }^{[19]}$. 


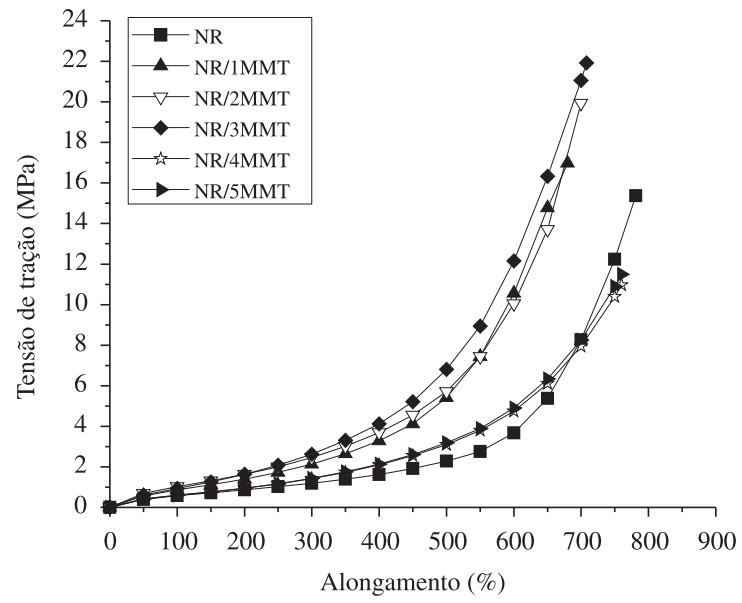

(a)

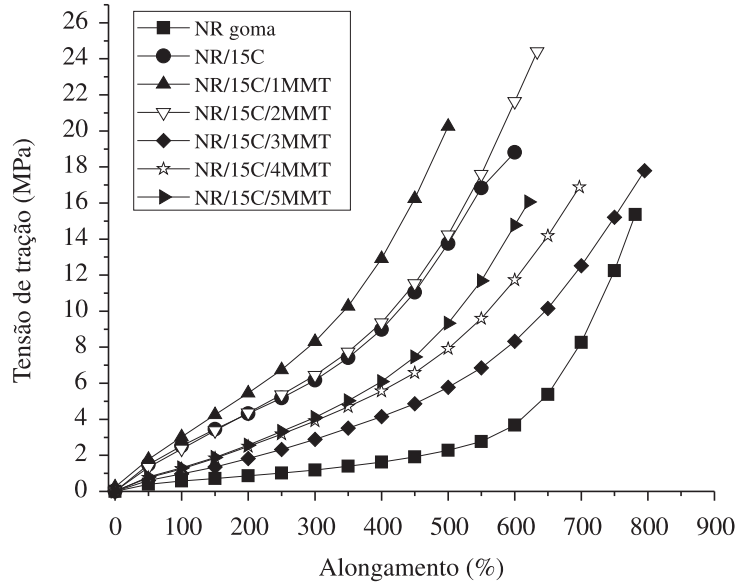

(b)

Figura 4. Tensão de tração dos nanocompósitos de (a) borracha natural com montmorilonita e (b) borracha natural com celulose II e montmorilonita.

Nas curvas apresentadas nas Figuras $4 \mathrm{a}$, b pode ser percebido que a adição de montmorilonita e celulose não impediram a cristalização sob tração dos nanocompósitos, indicando provavelmente uma interação física. Segundo Kraus ${ }^{[22]}$, as interações físicas são interações tipo van der Waals e são formadas por adsorção entre a superfície polimérica e a carga, contribuindo para o reforço da matriz. A cristalização induzida por tração das composições de borracha natural pode ser observada na curva tensão $\times$ deformação através do aumento da tensão e permanência ou pequena variação do alongamento. Durante o processo de cristalização sob tração ocorre a orientação das cadeias gerando o desenvolvimento de cristais de ordem tridimensional no material polimérico amorfo.

Para a composição goma pura o efeito da cristalização sob tensão pode ser observado nas Figuras $4 \mathrm{a}$ b após $600 \%$ de deformação, e para as outras composições, em deformações menores, em função do teor de carga.

Na Figura 5 estão os resultados de deformação permanente após a compressão (DPC) dos nanocompósitos de borracha natural. A composição de borracha sem carga apresentou a deformação por compressão menor que as demais composições ${ }^{[1,22]}$. As composições do sistema NR/MMT que apresentaram intercalação/esfoliação de montmorilonita, obtiveram menores resultados de deformação permanente, o mesmo acontecendo com a composição NR/15CEL/2MMT, corroborando os resultados de TEM e resistência à tração apresentados anteriormente.

As camadas de montmorilonita intercaladas/esfoliadas no sistema NR/CEL/MMT, encontram-se melhor dispersas na matriz elastomérica, permitindo que haja um confinamento e melhor distribuição das cadeias de borracha juntamente com a celulose, entre estas camadas. Esta forma de disposição da matriz e da celulose entre as camadas de argila permite com que a relaxação das cadeias de borracha após cessada a força de compressão aplicada, fosse mais efetiva, gerando melhor recuperação à deformação, e consequentemente, menor deformação permanente. A melhor dispersão de camadas permite que a energia absorvida durante a aplicação da força seja liberada de forma mais efetiva durante a relaxação das cadeias. Os agregados de carga presentes no sistema absorvem a energia com maior intensidade que a matriz elastomérica durante o ensaio, provocando a redução da relaxação das cadeias e da capacidade de recuperação elástica.

$\mathrm{O}$ uso da montmorilonita in natura, no sistema NR/CEL utilizado neste trabalho, promoveu o aumento da capacidade de recuperação à deformação permanente após a compressão dos nanocompósitos desenvolvidos.

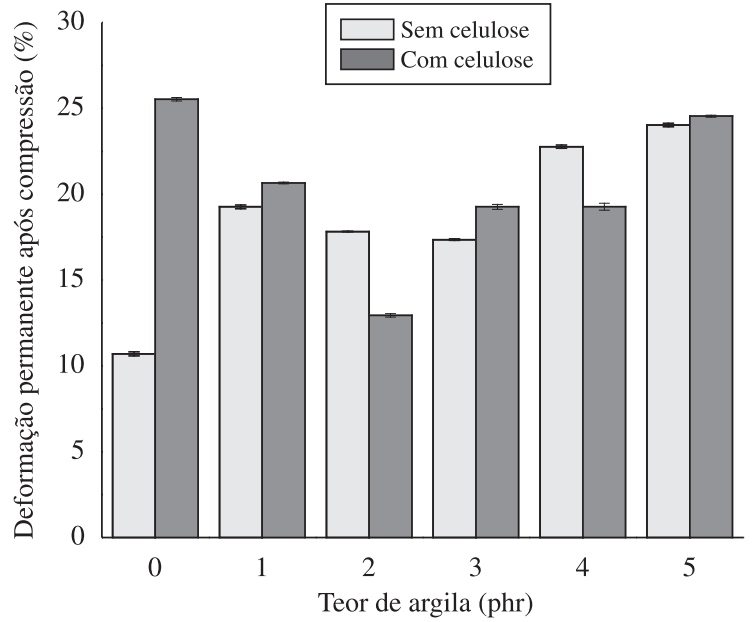

Figura 5. Deformação permanente após a compressão dos nanocompósitos de borracha natural com/sem celulose II e montmorilonita.

Na Figura 6 estão apresentados os resultados de resiliência dos nanocompósitos de borracha natural. A resiliência é função do módulo dinâmico e do atrito interno da borracha, e muito sensível a mudanças de temperatura. De acordo com a literatura ${ }^{[23,24]}$, a resiliência diminui com o aumento do teor de cargas e da densidade de ligações cruzadas formadas, aumentando a rigidez da matriz elastomérica. $\mathrm{O}$ desvio do estado elástico ideal pode ser obtido a partir da Equação 2 ${ }^{[1,22-24]}$ :

$$
R=\frac{[\text { energia queretorna do vulcanizado }]}{[\text { energia fornecida ao vulcanizado }]} \times 100 \%
$$

onde $\mathrm{R}$ é a resiliência, expressa em porcentagem. A partir desta expressão é possível quantificar a histerese $(\mathrm{H})$ que é a perda de energia que ocorre sob forma de calor e pode ser obtida a partir da Equação $3^{[1,22-24]}$.

$$
\mathrm{H}=100-\mathrm{R}
$$

Fatores que restringem a mobilidade dos segmentos da cadeia entre as ligações cruzadas, como a adição de carga, irá resultar em redução da flexibilidade, pois a cadeia não poderá mais retornar com a capacidade anterior. Com isso, parte da energia fornecida será dissipada na forma de calor diminuindo a resiliência do material.

De acordo com a Figura 6, a adição crescente de argila a matriz NR, sem celulose, provocou a redução da resiliência até o teor limite de 3 phr e após este teor, ocorreu o aumento da resiliência. A 


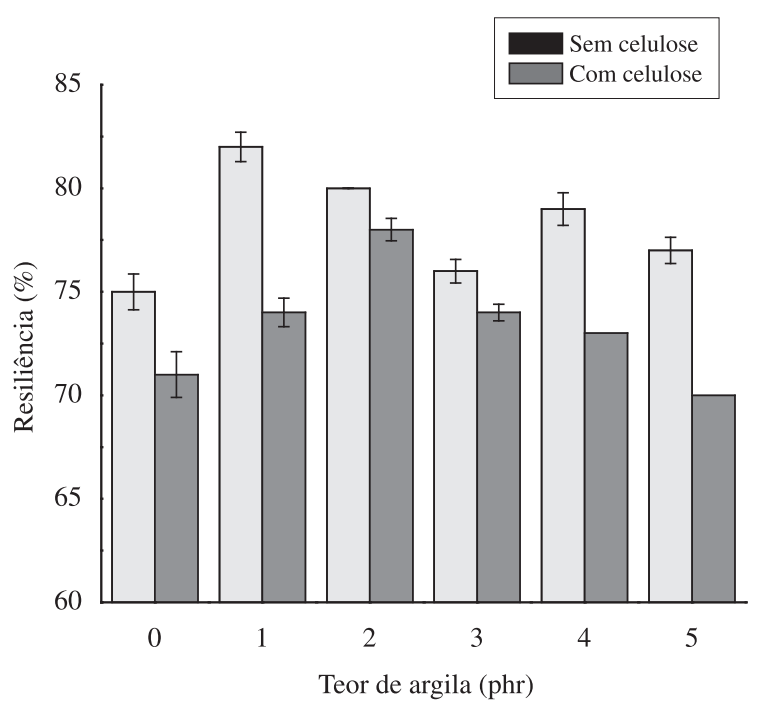

Figura 6. Resiliência dos nanocompósitos de borracha natural com/sem celulose II e montmorilonita.

intercalação/esfoliação das camadas de montmorilonita promoveu o aumentou da rigidez com consequente redução da resiliência, devido ao confinamento das cadeias de borracha entre as lamelas de argila, restringindo a mobilidade dos seguimentos da cadeia.

A formação de domínios de agregados de carga, verificados nos teores acima de $3 \mathrm{phr}$, podem estar contribuindo para o aumento da mobilidade das cadeias, provocando o aumento da resiliência. $\mathrm{O}$ aumento da resiliência também pode estar sendo provocado pela absorção de energia pelos aglomerados de carga. De acordo com Wang ${ }^{[14]}$, em um sistema polimérico, a rede de carga é o fator dominante na determinação da histerese no vulcanizado. A formação de uma rede de carga causa aumento no volume efetivo de carga fazendo com que a borracha permaneça presa nos aglomerados. A presença da celulose na matriz NR que aumenta a rigidez do nanocompósito causa redução da resiliência, porém a adição crescente de argila no sistema NR/CEL provocou o aumento da resiliência até o teor de $2 \mathrm{phr}$ de MMT, onde as camadas encontram-se intercaladas/esfoliadas ${ }^{[16]}$.

\section{Conclusão}

A técnica de co-coagulação do látex de borracha natural com xantato de celulose e suspensão aquosa de argilo mineral escolhida para o desenvolvimento deste trabalho mostrou-se efetiva e viável para a obtenção de nanocompósitos, como pode ser observado por TEM. O processo utilizado para intercalação/esfoliação da montmorilonita foi efetivo e simples e pode ser industrialmente proposto. A adição da montmorilonita in natura como carga no sistema NR/CEL promoveu a formação de interações entre a argila intercaladas/esfoliadas e o sistema NR/CEL. O efeito da intercalação/esfoliação da argila promoveu um aumento nas propriedades mecânicas estudadas. $\mathrm{O}$ aumento na viscosidade Mooney e a redução na capacidade de relaxação indicam boa interação entre a montmorilonita e o sistema NR/CEL.

\section{Agradecimentos}

Os autores agradecem ao apoio financeiro do Conselho Nacional de Desenvolvimento Científico e Tecnológico (CNPq), a Nitriflex Indústria e Comércio pela análise de viscosidade e relaxação Mooney, a Vicunha Têxtil pela doação do xantato de celulose, ao Instituto Nacional de Tecnologia pela doação da argila e o Comércio e Beneficiamento de Látex Talismã Ltda, pela doação do látex de borracha natural.

\section{Referências Bibliográficas}

1. Marck, J. E.; Erman, B. \& Eirich, F. R. - "Science and Technology of Rubber", Academic Press, New York (1994).

2. Alexandre, M. \& Dubois, P. - Mat. Sci. Eng., 28, p.1 (2000). http:// dx.doi.org/10.1016/S0927-796X(00)00012-7

3. Beecroft, L. L.; Johnen, N. A.; Ober, C. K. - Polym. Advan. Technol. 8, p.289 (1997). http://dx.doi.org/10.1002/(SICI)1099-1581(199705)8:5 $<289$ ::AID-PAT648>3.3.CO;2-V

4. Chang, J.-H.; Park, D.-K. - J. Polym. Sci.: Part B: Polym. Phys., 39, p.2581 (2001). http://dx.doi.org/10.1002/polb.10020

5. Lee, D. C.; Jang, L. W. - J. Appl. Polym. Sci, 68, p.1997 (1998). http:// dx.doi.org/10.1002/(SICI)1097-4628(19980620)68:12<1997::AIDAPP14>3.0.CO;2-P

6. Simon, I.; Glaser, L.; Scheraga, H. A. \& Manley, R. S. J. - Macromol., 21, p.983 (1988). http://dx.doi.org/10.1021/ma00182a024

7. Hon, D. N. S. - Polym. News, 13, p.134 (1988).

8. Mano, E. B.; Nunes, R. C. R. \& Cunha Lima, L. C. O. - "Processo para a Fabricação de Dispersões Homogêneas de Celulose e seus deri-vados em Elastômeros", Patente C08L1/22, n.7502614, abr. 1975.

9. De Carvalho Peres, A. C.; Nunes, R. C. R. \& Visconte, L. L.Y. - "Processo de obtenção de composições homogêneas de celulose, seus derivados e elastômeros, e suas composições", Patente PI 0105116-4 (2001) e CI 0105116-4 (2005).

10. Lapa, V. L. C.; Nunes, R. C. R. \& Visconte, L. L. Y. - Polym. Bull., 60, p.281 (2008).

11. Reis-Nunes, R. C.; Compañ, V. \& Riande, E. - J. Polym. Sci. Polym. Phys., 38, p.393 (2000). http://dx.doi.org/10.1002/(SICI)1099-0488(2 0000201)38:3<393::AID-POLB6>3.0.CO;2-K

12. Andrio, A.; Compañ, V.; Reis-Nunes, R. C.; López, M. L. \& Riande, E. - J. Membrane Sci., 178, p.65 (2000). http://dx.doi.org/10.1016/ S0376-7388(00)00476-2

13. Arroyo, M.; Lopez-Manchado, M. A. \& Herrero, B. - Polymer, 44, p.2447 (2003). http://dx.doi.org/10.1016/S0032-3861(03)00090-9

14. Wang, Y.; Zhang, L.; Tang, C. \& Yu, D. - J. Appl. Polym. Sci., 78, p.1879 (2000). http://dx.doi.org/10.1002/1097-4628(20001209)78:11 $<1879::$ AID-APP50>3.0.CO;2-1

15. Wu, Y. P.; Jia, Q. X.; Yu, D. S. \& Zhang, L. Q. - J. Appl. Polym. Sci., 89, p.3855 (2003). http://dx.doi.org/10.1002/app.12568

16. Mariano, R. M.; Picciani, P. H. S.; Nunes, R. C. R. \& Visconte, L. L. Y. - J. Appl. Polym. Sci., 120, p.458 (2011). http://dx.doi.org/10.1002/ app.33159

17. Madhusoodanan, K. N. \& Varguese, S. - J. Appl. Polym. Sci., 102, p.2537 (2006). http://dx.doi.org/10.1002/app.24680

18. Wu, Y. P. ; Wang, Y. Q.; Zhang, H. F.; Wang, Y. Z.; Yu, D. S.; Zhang, L. Q. \& Yang, J. - Compos. Sci. Technol., 65, p.1195 (2005). http://dx.doi. org/10.1016/j.compscitech.2004.11.016

19. Vu, Y. T.; Mark, J. E.; Pham, L. Y. H. \& Engelhardt, M. - J. Appl. Polym. Sci., 82, p.1391 (2001). http://dx.doi.org/10.1002/app.1976

20. Sombatsompop, N. - Polym. Plast. Technol. Eng., 37, p.19 (1998). http://dx.doi.org/10.1080/03602559808006910

21. Carretero-González, J. - Macromol., 41, p.6763 (2008).

22. Kraus, G. - "Reinforcement of Elastomers", Interscience Publishers, New York (1965).

23. Boonstra, B. B. \& Taylor, G. L. - Rubber Chem. Technol., 38, p.943 (1965). http://dx.doi.org/10.5254/1.3535712

24. Lorenz, O. \& Parks, C. R. - J. Polym. Sci., 50, p.299 (1961). http:// dx.doi.org/10.1002/pol.1961.1205015404 